\title{
Announcements and Reports
}

\section{Amtliche Mitteilungen}

Berichtszeitraum 22. Juli 2013 bis 19. August 2013

\section{Bereich Lebensmittel}

\section{(a)}

Ausnahmegenehmigung nach $\S 68$ Abs. 1 und 2 Nr. 1 des LFGB für die Einfuhr und das Inverkehrbringen

- von getrockneten Lindenblüten, die Rückstände bis zu $0,1 \mathrm{mg} / \mathrm{kg}$ DEET enthalten (Bekanntmachung des BVL 101.11257.0.0004 vom 09.08.2013; GMBl 2012, Nr. 47, S. 951). Es handelt sich um ein Produkt der Martin Bauer GmbH \& Co. KG, 91487 Vestenbergsgreuth.

(b)

Ausnahmegenehmigung nach $\S 68$ Abs. 1 und 2 Nr. 1 des LFGB für das Herstellen und Inverkehrbringen

- von jodiertem Speisesalz mit Zusatz von Kaliumfluorid (Bekanntmachung des BVL 101.11251.0.0012 vom 22.07.2013; GMBl im Druck). Es handelt sich um ein Produkt der Triton Resorces GmbH, 40212 Düsseldorf.

(c)

Änderung und Verlängerung einer

Ausnahmegenehmigung nach § 68 Abs. 1 und 2 Nr. 1 des LFGB für das Herstellen und Inverkehrbringen

- eines Nahrungsergänzungsmittels mit Zusatz von L-Carnitin-L-Tartrat (Bekanntmachung des BVL 101.222.8140.3.2298 vom 19.08.2013; GMBl im Druck). Es handelt sich um ein Produkt der ascopharm $\mathrm{GmbH}$ und der revoMed $\mathrm{GmbH}$, Bruchanger 6, 38855 Wernigerode.

- von vier angereicherten proteinhaltigen Getränkepulvern in verschiedenen Geschmacksrichtungen mit Zusatz von L-Glutamin (Bekanntmachung des BVL 101.222.8140.3.2401 vom 05.08.2013; GMBl im Druck). Es handelt sich um ein Produkt der Nutrichem Diät + Pharma GmbH, 91154 Roth.

\section{(d)}

Allgemeinverfügung gemäß § 54 LFGB über das Verbringen in die Bundesrepublik Deutschland und das Inverkehrbringen

- von Frühstückscerealien mit Zusatz von Vitamin D und Eisen (Bekanntmachung des BVL 101.11251.0.0015 vom 12.08.2013; GMBl im Druck).

- eines Nahrungsergänzungsmittels mit Zusatz von L-Arginin und L-Citrullin (Bekanntmachung des BVL 101.222.8140.3.2350 vom 19.08.2013; GMBl im Druck).

\section{Bereich Tierarzneimittel}

Die Bekanntmachung über die Zulassung von Tierarzneimitteln gemäß § 34 des Arzneimittelgesetzes in der Fassung der Bekanntmachung vom 12. Dezember 2005 (BGBl. I S. 3394) sowie gemäß $\S 6$ der Verordnung über homöopathische Arzneimittel vom 15. März 1978 (BGBl I S. 401) finden Sie auf der Homepage des BVL unter der Rubrik Tierarzneimittel: http://www.bvl.bund.de/bekanntmachung_tierarzneimittel. 